\title{
La coloscopie, cœur de notre métier
}

\section{Colonoscopy, the essence of our work}

\author{
G. Lesur \\ (C) Springer-Verlag France 2013
}

La coloscopie est sans aucun doute au cœur de notre métier. Elle l'est de par les chiffres (plus d'un million d'examens pratiqués chaque année) mais aussi de par la fréquence du cancer colorectal dans notre pays avec 40000 nouveaux cas et 17500 décès en 2010. Il est prouvé que les tests de recherche de sang dans les selles réduisent la mortalité et sans doute l'incidence du cancer colorectal. Mais on connaît les limites diagnostiques des tests actuels (sans parler des taux de participation très insuffisants puisque de l'ordre de $30 \%$ en moyenne), et on espère voir enfin commercialiser les tests immunologiques dont les performances sont meilleures. Le dépistage du cancer colorectal, au pronostic tellement meilleur si le diagnostic est précoce, repose aussi sur la coloscopie, autre pierre angulaire de la politique de prévention. Car il a également été montré que la coloscopie réduisait l'incidence et la mortalité du cancer colorectal même si cet effet n'est pas démontré pour les cancers du côlon droit.

On sait depuis plusieurs années que le taux de détections des polypes par coloscopie varie beaucoup d'un centre à l'autre et dans un même centre d'un opérateur à l'autre. Il a également été montré en 2006 qu'un temps de retrait de l'endoscope plus long permettait de trouver plus de lésions coliques. Une préparation de qualité est un élément majeur du succès de la coloscopie, et c'est à nous de prendre le temps d'expliquer en détail ses modalités pratiques et son importance. Utiliser des artifices techniques, tels un coloscope grand angle ou faire la coloscopie avec un cap sur l'endoscope, ne semble pas apporter de gain diagnostique. De même, il ne semble pas que la chromoendoscopie électronique améliore le taux de détection des adénomes. En revanche, il est montré que la haute définition augmente le taux de détection des lésions coliques. Il en est de même de la chromoendoscopie à l'indigo carmin, en particulier pour les adénomes festonnés. Et le commentaire d'article à la fin de ce numéro (p. 224) montre aussi qu'un temps de retrait élevé augmente le taux de détection de ces redoutables polypes festonnés du côlon proximal. Mais le temps de retrait n'explique pas tout, encore faut-il apprendre à trouver et à reconnaître ces lésions si souvent difficiles à voir. Le bénéfice à attendre sera donc certainement plus important chez des opérateurs exercés.

En d'autres termes, plus que jamais, il nous faut faire des coloscopies dans de bonnes conditions de préparation, avec des endoscopes modernes (voir l'article Recommandations de la Société française d'endoscopie digestive pour l'organisation et le fonctionnement d'un plateau technique en endoscopie digestive dans ce numéro, p. 198), prendre son temps au retrait de l'endoscope, notamment dans le côlon proximal, et utiliser l'indigo carmin au moins dans des côlons proximaux présumés normaux pour trouver plus de lésions festonnées. Il nous faut également mesurer notre taux de détection d'adénomes et mesurer son évolution dans le temps en cherchant à le faire croître. C'est à ce prix seulement que nous rendrons le plus grand service possible à tous et à chacun des hommes et femmes qui nous sont confiés pour une coloscopie. À un moment où l'incidence du cancer colorectal dans notre pays ne cesse de grimper, il nous faut impérativement tout faire pour casser cette courbe. L'exemple de la mortalité sur la route, en baisse spectaculaire ces dernières années, montre qu'une volonté forte est capable d'obtenir des résultats spectaculaires. À nous, acteurs de premier plan, de faire de même pour le cancer colorectal.
G. Lesur $(\bowtie)$

Hôpital Ambroise-Paré, F-92100 Boulogne, France

e-mail : gilles.lesur@apr.aphp.fr 